\title{
Immunity in Tuberculosis
}

\author{
Sotiros D. Chaparas, Director \\ Mycobacterial and Fungal Antigens Branch \\ Bureau of Biologics, Food and Drug Administration \\ Bethesda, MD 20205, USA
}

\begin{abstract}
Mycobacteria which produce disease in man are listed in Table 1. Tuberculosis is the most important of these diseases.
\end{abstract}

Table 1. Mycobacteria in Human Disease

\begin{tabular}{|c|c|c|}
\hline Species & Runyon Group & Disease \\
\hline $\begin{array}{l}\text { M. tuberculosis-africa- } \\
\text { num-bovis complex }\end{array}$ & & $\begin{array}{l}1^{\circ} \text { Pulmonary; may become systemic; } \\
\text { local }\end{array}$ \\
\hline M. leprae & v & $\begin{array}{l}\text { Predeliction for skin, nerves, cooler } \\
\text { parts of body. May be widely distri- } \\
\text { buted to other parts of body, e.g. } \\
\text { liver, spleen }\end{array}$ \\
\hline $\begin{array}{l}M . \text { ulcerans } \\
M . \text { hemophilum }\end{array}$ & & $\begin{array}{l}\text { Skin and underlying tissue } \\
\text { Extrapulmonary }\end{array}$ \\
\hline
\end{tabular}

I . Photochromogens; pigment only on exposure to light

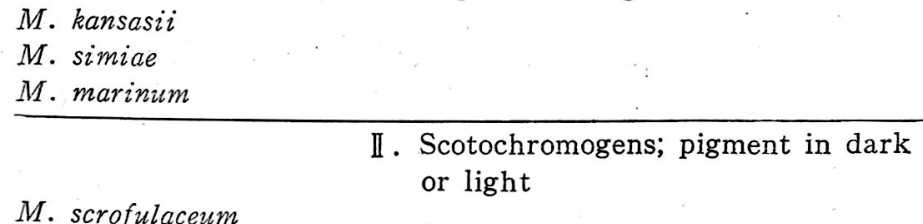

M. scrofulaceum

Similar to tuberculosis

Similar to tuberculosis

Skin, swimming pool granuloma

M. xenopi

Lymph node, esp. in children, rarely pulmonary

Pulmonary (contaminated water sources)

M. szulgal (scotochromo-

Pulmonary; lymph nodes genic at $37^{\circ} \mathrm{C}$ )

III. Nonphotochromogens; very little or no pigment in dark or light

M. avium-intracellulare

Pulmonary as in tuberculosis; lymph nodes in children. Often

opportunistic.

M. fortuitum-chelonei

IV. Rapid growers

Mature colonies by 3-5 days
Skin abscesses secondary to trauma; rarely pulmonary.

Opportunistic; contaminated heart valve transplants. 
Tuberculosis in Korea remains high relative to other nations in the, world. The effectiveness of BCG vaccine as prophylactic measure against tuberculosis has recently been questioned after analysis of results of a seven year trial in South India (1). The trial employed two dose levels of the French and Danish strains administered randomly and in a double-blind fashion to 260,000 subjects. Vaccination did not appear to convey any protection. There are factors with the trial which were not adequately considered and it is not proper to condemn
$B C G$ vaccines as inefficacious. The most glaring factor is the presence of nontuberculous mycobacteria in the study area (2). Nontuberculous mycobacteria sensitize and protect populations against tuberculosis (3, 4). BCG vaccination does not appear to add protection beyond that already induced by other mycobacterial infections(3). Antigenic sharing among species of mycobacteria is extensive as can be seen in figure 1. Of the numerous shared antigens a number are likely to be immunogenic.

Once a sensitivity to mycobacterial an-

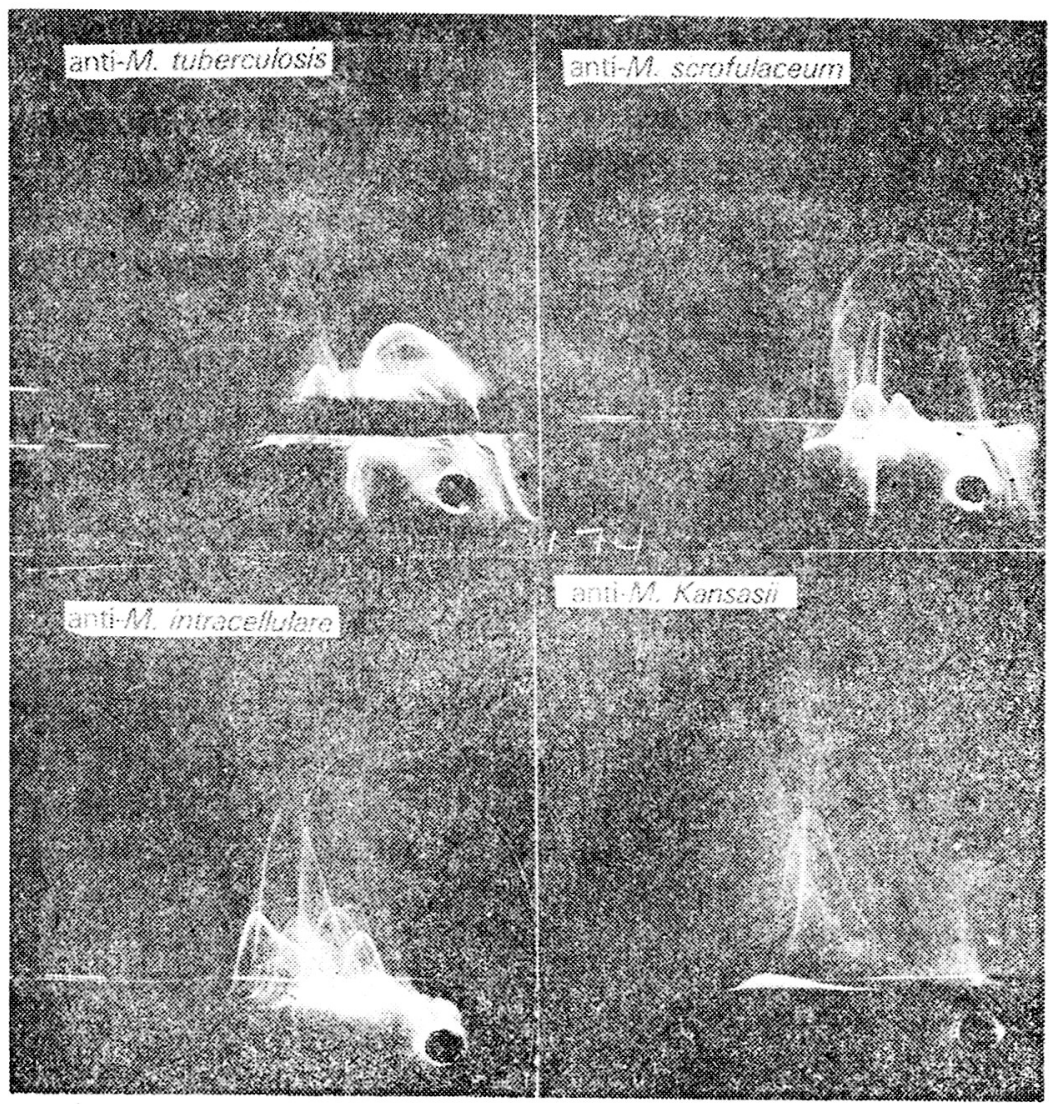

Fig. 1. Two-dimensional immunoelectrophoresis of $M$. tuberculosis extracts with various mycobacterial antisera. 
tigens is established the host can respond anamnestically to virulent infections with M. tuberculosis. Although it has been demonstrated that tuberculous infection may not be prevented, evidence has been presented that there exists an increased resistance to spread in BCG vaccinated persons (5). It is likely that avirulent tuberculous and nontuberculous infections similarly protect. The virulent infectious process itself evokes the anamnestic cellular immunity which limits the infection.

It should be remembered that immunity is relative and can be overcome if the infecting organism is very virulent and/or if the host's immunity becomes compromised. Thus many persons, with some immunity to tuberculosis. These individuals may nevertheless become superinfected with $M$. tuberculosis and may develop disease.

A positive skin test to tuberculin is hallmark of cellular immunity, the mechanism necessary to activate macrophage so that they can more effectively destroy tubercle

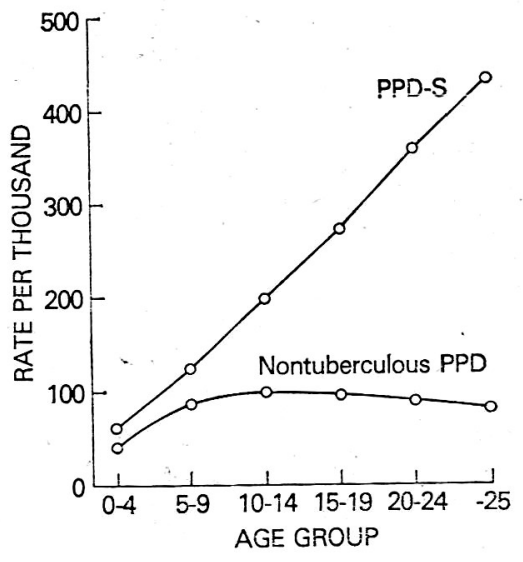

Fig. 2. Age Related Sensitivity to Tuberculin and Nontuberculous Antigens bacilli. Differential skin testing with antigens from selected species of mycobacteria can also reveal the natural history of infection to various mycobacterial species. In Haiti for example it has been shown that maximum sensitization to PPD preparations from nontuberculous mycobacteria is reached by age 10 . However, sensitivity to M. tuberculosis continues to increase well into adult ages (6) (Figure 2).

The Haitian situation is analogous to what might be expected in Korea. There seems to be little question that nontuberculous mycobacteria are endemic in Korea, indeed, diseases with these organisms have been reported. It is possible then that a considerable amount of immunity is conveyed by these infections.

Examination of distribution of reaction sizes to a standard dose of tujerculin can sometimes provide information as to the prevalence of nontuberculous infections in populations.

In countries with a low incidence of tuberculosis and nontuberculous infection, a normal bimodal distribution of reaction sizes is seen as in figure 3 panel A. In regions.

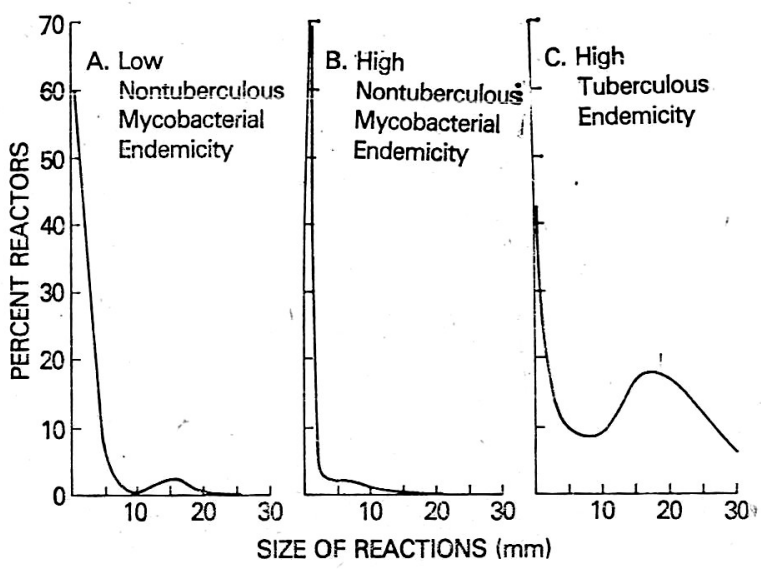

Fig. 3. Distribution of Skin Test Reaction sizes in Various Populations 
with significant infection rate with norituberculous mycobacteria, the bimodal distribution is lost and reactions between $5 \mathrm{~mm}$ and $7 \mathrm{~mm}$ occur more frequently (Fig. 3, panel B). However, when a high tuberculosis rate occurs, as seen in Korea, a bimodal distribution occurs with a higher baseline which masks reactions due to nontuberculous mycobacteria sensitivity (Fig. 3, panel C).

In order to determine of nontuberculous infections in Korea and their potential role as inducers of cross protection to $M$. tuberculosis, it may be necessary to determine the age specific infection rates with endemic species of mycobacteria, especially in very young children between 1 and 10 years of age. Since the tuberculosis infection rate is high, it is expected that with increasing age greater and greater portions of the population will become infected for the first time or superinfected with $M$. tuberculosis. Such analyses will permit a better understanding of the potential role of nontuberculous mycobacterial infections and of $B C G$ vaccination in protection against tubercu losis.

\section{References}

1. Tuberculosis Prevention Trial, Trial of BCG Vaccines in South India for tuberculosis prevention, Ind. J. Med. Res., 70, 349, 1979; Bull. Wld. Hlth. Org. 57, 819, 1979.

2. Narain, R., Krishamurthy, M.S., Anantharaman, D.S., Prevalence of nonspecific sensitivity in some parts of India, Ind. J. Med. Res. 63, 1099, 1975.

3. Edwards, L.B. and Palmer, C.E., Identification of the tuberculous-infected by skin tests, Ann. N.Y. Acad. Sci., 154, 140, 1968.

4. Comstock, G.W. and Webster, R.G. Tuberculosis studies in Muscogee County, Georgia, Am. Rev. Resp. Dis. 100, 839, 1969.

5. Sutherland, I., and Lindgren, I., The protective effect of BCG vaccination as indicated by autopsy studies, Tubercle, 60, 225, 1979.

6. Vandiviere, H.M., Melvin, I. G., Narain, R., Harris, W.D.M., and Chaparas, S.D., Profiles of skin test reactivity to antigens of various mycobacterial species in a human population and in experimental infections, Tubercle (in press). 